\title{
The Maximal Kirchhoff Index of Theta Shape Graph
}

\author{
Jinyu Zou ${ }^{1,2}$ and Haizhen Ren ${ }^{3+}$ \\ ${ }^{1}$ School of Computer, Qinghai Normal University, Xining, Qinghai 810008, China \\ ${ }^{2}$ Department of Basic Research, Qinghai University, Xining, Qinghai 810008, China \\ ${ }^{3}$ Department of Mathematics, Qinghai Normal University, Xining 810008, Qinghai, China
}

\begin{abstract}
The resistance distance between any two vertices of a connected graph $\mathrm{G}$ is defined as the effective resistance between them in the electrical network constructed from $G$ by replacing each edge of $G$ with unit resistor. The Kirchhoff index of a graph is a structure-descriptor based on resistance distance. The investigation on the Kirchhoff index of graph is an important topic in the theory of graph. It is difficult to implement some algorithms to compute resistance distance and Kirchhoff index in a graph from their computational complexity. Hence, it makes sense to find closed-form formulae or solve extreme problems for the Kirchhoff index. For the connected graphs whose cyclomatic number less than two, their resistance distances and the Kirchhoff indices have been described well. In this paper, we discuss the graphs with cyclomatic number two, by graph transformations the maximal Kirchhoff index and the corresponding graph in the theta shape graphs (a specified class of bicycle graphs) are obtained.
\end{abstract}

Keywords: electrical network, resistance distance, Kirchhoff index, theta shape graph.

\section{Introduction}

The graph $G$ considered here is simple undirected, with the vertex set $V(G)$ and edge set $E(G)$. We denote the number of the vertices of $G$ by $|V(G)|$, and the number of the edges of $G$ by $|E(G)|$. The distance between vertices $u$ and $v$ of graph $G$, denoted by $d(u, v)$, is the length of the shortest path between them. If e is an edge of a connected graph $\mathrm{G}$, we denoted by $\mathrm{G}$-e the graph obtained from $\mathrm{G}$ by deleting the edge e, and Gle the graph obtained by removing the edge e and identifying its two end-vertices.

On the basis of electrical network theory, Klein and Randic introduced the novel concept of resistance distance[1]. Let $\mathrm{G}$ be a connected graph with vertices set $v_{1}, v_{2}, \cdots, v_{n}$, they viewed a graph $\mathrm{G}$ as an electrical network $\mathrm{N}$ such that each edge of $\mathrm{G}$ is assumed to be a unit resistor, the resistance distance between vertices $v_{i}$ and $v_{j}$, denoted by $r\left(v_{i}, v_{j}\right)$ or $r_{i j}$, is defined to be the effective resistance between nodes $v_{i}$ and $v_{j}$ as computed with Ohm's law in N. The Kirchhoff index of G, defined as [1], $K f(G)=\sum_{i<} r_{i j}$, is the sum of resistance distances between all pairs of vertices in G. The famous Wiener index was denoted as W(G)[2], $W(G)=\sum_{i<i} d_{i j}$, which counts the sum of distances between pairs of vertices of G. Klein and Randic proved that $r_{i j} \leq d_{i j}$ and $K f(G) \leq W(G)$ with equality if and only if $\mathrm{G}$ is a tree[1]. Kirchhoff index is a structure-descriptor based on resistance distance. The investigation on the Kirchhoff index of graph is an important topic in the theory of graph. It is difficult to implement some algorithms to compute resistance distance and Kirchhoff index in a graph from their computational complexity. Hence, it makes sense to find closed-form formulae for the Kirchhoff index [1,3]. In present, to compute resistance distance, various methods have been developed, and relevant for resistance distance have been given for some classes of graphs, and some relevant indices to Kirchhoff index are discussed[3-16]. For the connected graphs whose cyclomatic number less than two, their resistance distances and the Kirchhoff indices have been described well. In this paper, we discuss the bicycle graphs, whose cyclomatic number is two.

+ Corresponding author. Tel.: +09716307622; fax: +09716307622.

E-mail address: haizhenr@126.com 
The bicycle graphs are connected graphs whose number of vertices is one more than the number of edges. The first and second classes of these graphs have been discussed, and their ordering relations and extreme graphs are obtained [11]. In this paper, we will pay attention to the third class of graphs, i.e. the theta shape graphs (as shown in Fig.1 (1)). We denote the theta shape graph with $\mathrm{n}$ vertices by $\Theta_{n} . \Theta_{n}^{t}$ denotes the theta shape graph with $n$ vertices, and the number of the vertices on the essential circles is t. In [16], We have investigated the ordering relations of the Kirchhoff index of the theta shape graphs and the minimal Kirchhoff index and the corresponding graph in this class of graphs are also discussed. In this paper, by the graph transformation, we find some ordering relations of Kirchhoff indices and discuss the maximal Kirchhoff index for this class of graphs.

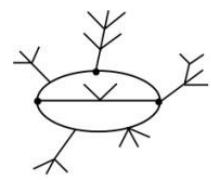

(1)

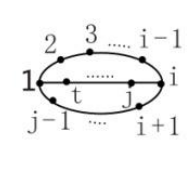

(2)

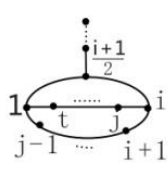

$\mathrm{G}_{1}$

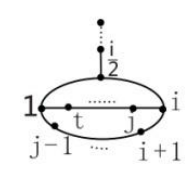

$\mathrm{G}_{2}$

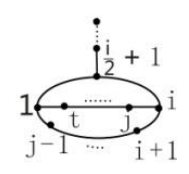

$G_{3}$

(3)
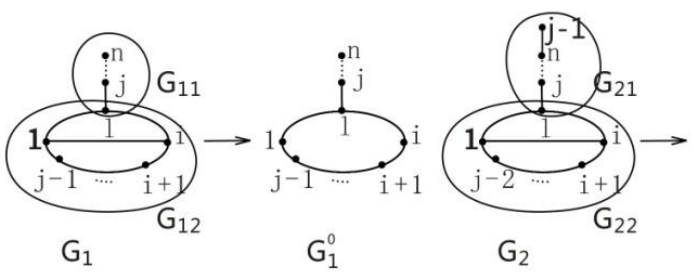

$\mathrm{G}_{2}$

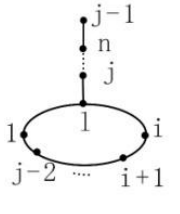

$\mathrm{G}_{2}^{0}$

(4)

Fig. 1: $\Theta_{n}, \Theta_{n}^{t}$ and their extreme graphs

The sum of the resistance distance from $v_{i}$ to other vertices of $\mathrm{G}$ is denoted by $\mathrm{Kf}_{v_{i}}(G)$. The following lemmas will be used in sequel:

Lemma 1.1([8]) For a general graph $\mathrm{G}, K f(G) \geq n-1$, the equality holds if and only if $\mathrm{G}$ is a complete graph.

Lemma 1.2([9]) For a graph $\mathrm{G}, K f(G) \leq\left(n^{3}-n\right) / 6$ with equality if and only if $\mathrm{G}$ is a path.

Lemma 1.3([10]) For a circulate graph $\mathrm{G}, n-1 \leq K f(G) \leq\left(n^{3}-n\right) / 12$, the first equality holds if and only if $\mathrm{G}$ is $K_{n}$ and the second does if and only if $\mathrm{G}$ is $C_{n}$.

Lemma 1.4([11]) Let $C_{n}$ be the cycle on $n \geq 3$ vertices, for any two vertices $v_{i}, v_{j} \in V\left(C_{n}\right)$ with $i<j$, by ohm's law, we have $r_{C_{n}}\left(v_{i}, v_{j}\right)=(j-i)(n+i-j) / n$.

Lemma 1.5([12]) Let $\mathrm{x}$ be a cut vertex of a connected graph $\mathrm{G}$ such that $\mathrm{G}-\mathrm{x}$ has exactly two branches $\mathrm{G}_{1}$ and $\mathrm{G}_{2}$. Let $G_{i}^{\prime}$ be the subgraph induced by $G_{i} \cup\{x\}(i=1,2)$. Then

$$
K f(G)=K f\left(G_{1}{ }^{\prime}\right)+K f\left(G_{2}{ }^{\prime}\right)+\left(\left|V\left(G_{1}{ }^{\prime}\right)\right|-1\right) K f_{x}\left(G_{2}{ }^{\prime}\right)+\left(\left|V\left(G_{2}{ }^{\prime}\right)\right|-1\right) K f_{x}\left(G_{1}{ }^{\prime}\right),
$$

Where $K f_{x}\left(G_{i}^{\prime}\right)=\sum_{v \in V\left(G_{i}^{\prime}\right)} r(x, v), i=1,2$.

Lemma 1.6([13]) Let $G^{\prime}$ be a connected graph with $e=i j$ being an edge, $G=G^{\prime}-e$, then for any $p, q \in V(G)\left(=V\left(G^{\prime}\right)\right)$

$$
r^{\prime}(p, q)=r(p, q)-\frac{[r(p, i)+r(q, j)-r(p, j)-r(q, i)]^{2}}{4[1+r(i, j)]}
$$

Lemma 1.7([13]) Let $G^{\prime}$ be a connected graph with $e=i j$ being an edge, $G=G^{\prime}-e$, then

$$
K f\left(G^{\prime}\right)=K f(G)-\frac{n \sum_{k=1}^{n}[r(i, k)-r(j, k)]^{2}-\left[\sum_{k=1}^{n} r(i, k)-\sum_{k=1}^{n} r(j, k)\right]^{2}}{4[1+r(i, j)]} .
$$

Lemma 1.8([13]) Let $\mathrm{G}$ be a connected graph with $e=i j$ being an edge. Let $G^{\prime}=G-e$ and $G^{*}=G \backslash e$. Then for any $p, q \in V(G), r(p, q)=[1-r(i, j)] r^{\prime}(p, q)+r(i, j) r^{*}(p, q)$. 


\section{Main Results}

\subsection{Two graph transformations and some ordering relations}

Transformation 1: The graph $G$ is a connected graph with vertex set $\left\{v_{1}, v_{2}, \cdots, v_{p}\right\}$, and each vertex $v_{i}(1 \leq i \leq p)$ with hanging tree $T_{i}$, without loss of generality, we suppose the tree $T_{i}$ with the maximal diameter q. We choose one path of length equal to the diameter of $T_{i}$, with the corresponding vertices $v_{i}, v_{i l}, v_{i 2}, \cdots, v_{i j}, \cdots, v_{i q}$. If each vertex $v_{i j}$ with hanging tree $T_{i}$, we denote such graph as $G_{1}$. If the tree $T_{i j}$ is adhered to $v_{i q}$, we have the graph $G_{2}$ (see Fig. 2(1)). We denote the set of vertices of $T_{i j} \backslash v_{i j}$ as $V_{1}$, the set $\left\{v_{i, j+1}, \cdots, v_{i q}\right\}$ as $V_{2}$.

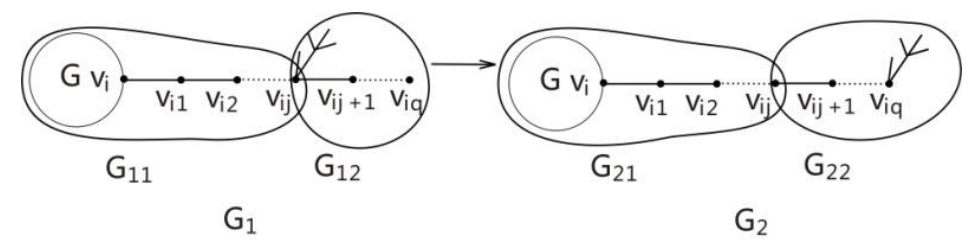

( 1 )

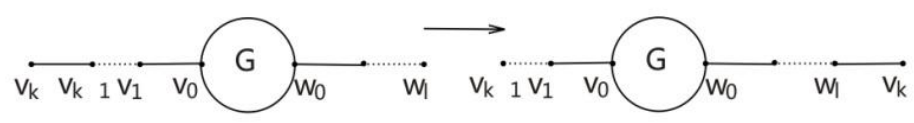

$\mathrm{G}_{1}$

$\mathrm{G}_{2}$

Fig. 2: Two graph transformations

Theorem 2.1 The graph $G_{1}$ and $G_{2}$ are such graphs given in Fig.2 (1), then $K f\left(G_{2}\right)>K f\left(G_{1}\right)$.

Proof. By Lemma 1.5, we have $K f\left(G_{1}\right)=K f\left(G_{11}\right)+K f\left(G_{12}\right)+\left(\left|V\left(G_{11}\right)\right|-1\right) K f_{v_{i j}}\left(G_{12}\right)+\left(\left|V\left(G_{12}\right)\right|-1\right) K f_{v_{i j}}\left(G_{11}\right)$ and

$$
K f\left(G_{2}\right)=K f\left(G_{21}\right)+K f\left(G_{22}\right)+\left(\left|V\left(G_{21}\right)\right|-1\right) K f_{v_{j i}}\left(G_{22}\right)+\left(\left|V\left(G_{22}\right)\right|-1\right) K f_{v_{j j}}\left(G_{21}\right) .
$$

As $G_{11} \cong G_{21}, G_{12} \cong G_{22}, K f\left(G_{11}\right)=K f\left(G_{21}\right), K f\left(G_{12}\right)=K f\left(G_{22}\right)$. And $K f_{v_{i j}}\left(G_{11}\right)=K f_{v_{i j}}\left(G_{21}\right),\left|V\left(G_{11}\right)\right|=\left|V\left(G_{21}\right)\right|$, $\left|V\left(G_{12}\right)\right|=\left|V\left(G_{22}\right)\right|$. So we are sufficient to compare $K f_{v_{i j}}\left(G_{12}\right)$ with $K f_{v_{v j}}\left(G_{22}\right)$,

$$
\begin{gathered}
K f_{v_{i j}\left(G_{12}\right)}=\sum_{v \in V_{1}} r_{G_{12}}\left(v_{i j}, v\right)+\sum_{v \in V_{2}} r_{G_{12}}\left(v_{i j}, v\right), \\
K f_{v_{i j}}\left(G_{22}\right)=\sum_{v \in V_{1}} r_{G_{22}}\left(v_{i j}, v\right)+\sum_{v \in V_{2}} r_{G_{22}}\left(v_{i j}, v\right)=\sum_{v \in V_{1}}\left[d\left(v_{i j}, v_{i q}\right)+r_{G_{12}}\left(v_{i j}, v\right)\right]+\sum_{v \in V_{2}} r_{G_{22}}\left(v_{i j}, v\right)
\end{gathered}
$$

As $d\left(v_{i j}, v_{i q}\right)>0$, then $K f_{v_{i j}}\left(G_{12}\right)<K f_{v_{i j}}\left(G_{22}\right)$. The result is obtained.

Transformation 2: The graph G with $|V(G)| \geq 3$ and we choose two vertices of $G$, for example $v_{0}$ and $w_{0}$ with $d\left(v_{0}, w_{0}\right) \geq 2$, there is an automorphism $\Phi$ of $G$ such that $\Phi\left(w_{0}\right)=\Phi\left(v_{0}\right)$, then for any $u \in V(G), r\left(v_{0}, u\right)=r\left(w_{0}, u\right) . P_{k}$ and $P_{l}$ are two paths with length $k$ and $l$ respectively, and $k \leq l$. The graph $G_{1}$ is obtained from $G$ if $v_{0}$ and $w_{0}$ with hanging path $P_{k}$ and $P_{l}$ respectively, and $G_{2}$ is obtained from $G$ if $v_{0}$ and $w_{0}$ with hanging path $P_{k-1}$ and $P_{l+1}$ respectively.(see Fig.2(2))

Theorem 2.2 The graph $G_{1}$ and $G_{2}$ are such graphs given in Fig.2 (2), then $K f\left(G_{1}\right)<K f\left(G_{2}\right)$.

Proof. By Lemma 1.5,

$$
\begin{aligned}
& K f_{v_{k}}\left(G_{1}\right)=\sum_{u \in V_{F_{k}}} r\left(v_{k}, u\right)+\sum_{u \in V(G)} r\left(v_{k}, u\right)+\sum_{u \in V_{A}} r\left(v_{k}, u\right)=\frac{k(k-1)}{2}+\sum_{u \in V(G)}\left[k+r\left(v_{0}, u\right)\right]+\sum_{i=1}^{l}\left[k+r\left(v_{0}, w_{0}\right)+i\right] \\
& =\frac{k(k-1)}{2}+k|V(G)|+K f_{v_{0}}(G)+k l+\frac{l(l+1)}{2}+l r\left(v_{0}, w_{0}\right) \\
& K f_{w_{l}}\left(G_{1}\right)=\sum_{u \in V_{F_{k}}} r\left(w_{l}, u\right)+\sum_{u \in V(G)} r\left(v_{k}, u\right)+\sum_{u \in V_{h}} r\left(w_{l}, u\right)=\frac{l(l-1)}{2}+\sum_{u \in V(G)}\left[l+r\left(w_{0}, u\right)\right]+\sum_{i=1}^{k}\left[l+r\left(v_{0}, w_{0}\right)+i\right] \\
& =\frac{l(l-1)}{2}+l|V(G)|+K f_{w_{0}}(G)+k l+\frac{k(k+1)}{2}+k r\left(w_{0}, v_{0}\right)
\end{aligned}
$$

So $K f_{w_{i}}\left(G_{1}\right)-K f_{v_{k}}\left(G_{1}\right)=(l-k)\left(|V(G)|-r\left(v_{0}, w_{0}\right)-1\right)$. Since $|V(G)|-1 \geq d\left(v_{0}, w_{0}\right) \geq r\left(v_{0}, w_{0}\right)$, and $l-k \geq 0$.

Then $K f_{v_{k}}\left(G_{1}\right) \leq K f_{w_{i}}\left(G_{1}\right)$. Thus $K f_{v_{k}}\left(G_{2}\right)=K f_{w_{i}}\left(G_{1}\right)-r_{G_{1}}\left(w_{l}, v_{k}\right)+|V(G)|-1>K f_{w_{i}}\left(G_{1}\right)$. Therefore $K f_{v_{k}}\left(G_{2}\right)>K f_{v_{k}}\left(G_{1}\right)$. Since $K f\left(G_{2}\right)-K f\left(G_{1}\right)=K f_{v_{k}}\left(G_{2}\right)-K f_{v_{k}}\left(G_{1}\right)$, then we get $K f\left(G_{2}\right)>K f\left(G_{1}\right)$. 
Theorem 2.3 $G_{0} \in \Theta_{n}$ is the graph with the maximal Kirchhoff index, then there must be a vertex of $G_{0}$ with only one vertex hanging path.

Proof. The result can be obtained by Theorem 2.1 and Theorem 2.2 easily.

\section{Theorem 2.4}

The graph $G$ is a graph depicted in Fig.1(2), $i<j<\mathrm{t}, G_{1}=G-e_{l, l+1}, G_{2}=G \backslash e_{l, l+1}(1 \leq l \leq i-1)$, then $K f_{\frac{v+l}{2}}(\mathrm{G})$ is the maximal amongst all vertices of $G$ when $i$ is odd, $K f_{v_{i}}(\mathrm{G})=K f_{v_{i+1}}(\mathrm{G})$ are the maximal when $i$ is even.

Proof. $\forall q \in V(G)$, by Lemma $1.8 r_{G}(l, q)=\left[1-r_{G}(l, l+1)\right] r_{G_{1}}(l, q)+r_{G}(l, l+1) r_{G_{2}}(l, q)$,

$$
r_{G}(l+1, q)=\left[1-r_{G}(l, l+1)\right] r_{G_{1}}(l+1, q)+r_{G}(l, l+1) r_{G_{2}}(l+1, q)
$$

If we want to compare $K f_{v_{l}}(G)$ with $K f_{v_{t+1}}(G)$, we are sufficient to compare $K f_{v_{l}}\left(G_{1}\right)$ with $K f_{v_{t+1}}\left(G_{1}\right)$. And $K f_{v_{l}}\left(G_{1}\right)=\sum_{1 \leq q \leq t, q \neq l} r_{G_{1}}(l, q), K f_{v_{l+1}}\left(G_{1}\right)=\sum_{1 \leq q \leq t, q \neq l+1} r_{G_{1}}(l+1, q)$, so if $1 \leq l \leq\left[\frac{i}{2}\right], K f_{v_{l}}\left(G_{1}\right) \leq K f_{v_{l+1}}\left(G_{1}\right), K f_{v_{l}}(G) \leq K f_{v_{t+1}}(G)$; if $\left[\frac{i}{2}\right]<l \leq i, K f_{v_{l}}\left(G_{1}\right) \geq K f_{v_{l+1}}\left(G_{1}\right), K f_{v_{l}}(G) \geq K f_{v_{l+1}}(G)$. Thus we complete the proof.

Theorem 2.5 If $G_{0} \in \Theta_{n}^{t}$ is the graph with the maximal Kirchhoff index, then $G_{0} \cong G_{1}$ when $i$ is odd, and $G_{0} \cong G_{2}, G_{3}$ when $i$ is even.(see Fig.1(3)).

Proof. The result is obtained from Theorem 2.4.

\subsection{The maximal Kirchhoff index and the corresponding graph}

In this section we consider $G \in \Theta_{n}^{t}$ such that $t=j-1, i$ and $j$ are both odd,

\section{Theorem 2.6}

The graph $G_{1} \in \Theta_{n}^{j-1}$ and $v_{i+1}$ with hanging path $P_{n-j+1}$, the graph $G_{2} \in \Theta_{n}^{j-2}$ and $v_{i+1}$ with hanging path $P_{n-j+2}$, then $K f\left(G_{1}\right)<K f\left(G_{2}\right)$. (see Fig.1(4))

Proof. Suppose $G_{1}^{\prime} \cong G_{1}-e_{1 i}$ and $G_{2}^{\prime} \cong G_{2}-e_{1 i}$, by Lemma 1.7 ,

$$
\begin{gathered}
K f\left(G_{1}\right)=K f\left(G_{1}^{\prime}\right)-\frac{n \sum_{k=1}^{n}\left[r_{G_{1}^{\prime}}(1, k)-r_{G_{1}^{\prime}}(i, k)\right]^{2}-\left[\sum_{k=1}^{n} r_{G_{1}^{\prime}}(1, k)-\sum_{k=1}^{n} r_{G_{1}^{\prime}}(i, k)\right]^{2}}{4\left[1+r_{G_{1}^{\prime}}(1, \mathrm{i})\right]} . \\
K f\left(G_{2}\right)=K f\left(G_{2}^{\prime}\right)-\frac{n \sum_{k=1}^{n}\left[r_{G_{2}^{\prime}}(1, k)-r_{G_{2}^{\prime}}(i, k)\right]^{2}-\left[\sum_{k=1}^{n} r_{G_{2}^{\prime}}(1, k)-\sum_{k=1}^{n} r_{G_{2}^{\prime}}(i, k)\right]^{2}}{4\left[1+r_{G_{2}^{\prime}}(1, \mathrm{i})\right]} .
\end{gathered}
$$

As $\sum_{k=1}^{n} r_{G_{1}^{\prime}}(1, k)=\sum_{k=1}^{j-1} r_{G_{1}^{\prime}}(1, k)+\sum_{k=j}^{n} r_{G_{1}^{\prime}}(1, k)=\sum_{k=1}^{j-1} r_{G_{1}^{\prime}}(1, k)+\sum_{k=j}^{n}\left(\mathrm{~d}_{G_{1}^{\prime}}(l, k)+r_{G_{1}^{\prime}}(l, 1)\right)$,

$$
\sum_{k=1}^{n} r_{G_{1}^{\prime}}(\mathrm{i}, k)=\sum_{k=1}^{j-1} r_{G_{1}^{\prime}}(\mathrm{i}, k)+\sum_{k=j}^{n}\left(\mathrm{~d}_{G_{1}^{\prime}}(l, k)+r_{G_{1}^{\prime}}(l, \mathrm{i})\right) \text {, and } r_{G_{1}^{\prime}}(l, 1)=r_{G_{1}^{\prime}}(l, i), \sum_{k=1}^{j-1} r_{G_{1}^{\prime}}(1, k)=\sum_{k=1}^{j-1} r_{G^{\prime}}(i, k),
$$

so $\sum_{k=1}^{n} r_{G_{1}^{\prime}}(1, k)=\sum_{k=1}^{n} r_{G_{1}^{\prime}}(i, k)$. Similarly, $\sum_{k=1}^{n} r_{G_{2}^{\prime}}(1, k)=\sum_{k=1}^{n} r_{G_{2}^{\prime}}(i, k)$. As $G_{1}^{\prime}$ and $G_{2}^{\prime}$ are both bicycle graphs, by the result of [11], we have $K f\left(G_{1}^{\prime}\right)<K f\left(G_{2}^{\prime}\right)$. Thus,

$$
\frac{\sum_{k=1}^{n}\left[r_{G_{1}^{\prime}}(1, k)-r_{G_{1}^{\prime}}(i, k)\right]^{2}}{1+r_{G_{1}^{\prime}}(1, \mathrm{i})}-\frac{\sum_{k=1}^{n}\left[r_{G_{2}^{\prime}}(1, k)-r_{G_{2}^{\prime}}(i, k)\right]^{2}}{1+r_{G_{2}^{\prime}}(1, \mathrm{i})}=\frac{(i-1)^{2}(i+1)\left(i^{2}-2 i+3\right)-(i-1)^{2}\left(2 i^{2}-i+2\right) j+i(i-1)^{2} j^{2}}{3\left(1+i^{2}-i j\right)\left(1-i+i^{2}-i j\right)}
$$

As $i \geq 3, j \geq 5, j \geq i+2,1+i^{2}-i j \leq 1-2 i<0,1-i+i^{2}-i j \leq 1-3 i<0$, then $3\left(1+i^{2}-i j\right)\left(1-i+i^{2}-i j\right)>0$.

And suppose that the function $f(j)=(i-1)^{2}(i+1)\left(i^{2}-2 i+3\right)-(i-1)^{2}\left(2 i^{2}-i+2\right) j+i(i-1)^{2} j^{2}, i(i-1)^{2}>0$, $(i-1)^{2}(i+1)\left(i^{2}-2 i+3\right)>0,-(i-1)^{2}\left(2 i^{2}-i+2\right)<0$, so if $j \geq 5, f(j)>0$. Thus $K f\left(G_{1}\right)<K f\left(G_{2}\right)$.

\section{Conclusion}

We have studied the case that $i$ and $j$ are both odd and $t=j-1$ and the corresponding theta shape graph with the maximal Kirchhoff index is also obtained. Now we haven't good method to solve other cases, they are also open. 


\section{Acknowledgements}

This work is partially supported by the Qinghai Natural Science Foundation of China (Grant Nos. 2015ZJ-911, 2016-ZJ-775), Key Laboratory of IOT of Qinghai Province and the National Natural Science Foundation of China (Grant Nos. 11551003).

\section{References}

[1] D. J. Klein, M. Randic, Resistance distance, J. Math. Chem. 1993, 12, 81-95.

[2] H. Wiener, Structural determination of paraffin boiling points, J. Amer. Chem. Soc. 1947,69: 17-20.

[3] Y. J. Yang and D. J. Klein, A recursion formula for resistance distance and its applications, Discrete Appl. Math. 2013,161:2702-2715.

[4] S. B. Huang, J. Zhou, C. J. Bu, Some Results on Kirchhoff Index and Degree-Kirchhoff Index, MATCH Commun. Math. Comput. Chem. 2016,75 : 207-222.

[5] M. Bianchi, A. Cornaro, J. L. Palacios, J. M. Renom and A. Torriero, Revisiting Bounds for the Multiplicative Degree-Kirchhoff Index, MATCH Commun. Math. Comput. Chem. 2016,75 : 227-231.

[6] H. P. Zhang, Y. J. Yang and C. W. Li, Kirchhoff index of composite graphs, Discrete Appl. Math. 2009,157: 2918-2927.

[7] I. Lukovits, S. Nikolic, N. Trinajstic, Resistance distance in regular graphs, Int. J. Quantum Chem. 1999,71:217225.

[8] J. L. Palacios, Resistance distance in graphs and random walks, Int. J. Quantum Chem. 2001,81:29-33.

[9] H. P. Zhang and Y. J. Yang, Resistance distance and Kirchhoff index in circulate graphs, Int. J. Quantum Chem. 2007,107:330-339.

[10] S. B. Chen, Q. Chen, X. Cai and Z. J. Guo, Maximal Degree Resistance Distance of Unicycle Graphs, MATCH Commun. Math. Comput. Chem. 2016,75: 157-168.

[11] X. Y. Jiang, The Kirchhoff Index extreme of the bicycle graphs, The master's degree thesis, 2008.

[12] X. Gao, Y. Luo and W. Liu, Resistance distances and the Kirchhoff index in Cayley graphs, Discrete Appl. Math. 2011,139:2050-2057.

[13] H. P. Zhang, Y. J. Yang, Kirchhoff Index of Linear Hexagonal Chains, International Journal of Quantum Chemistry, 2008,138:503-512.

[14] R. C. Entringer, D. E. Jackson and D. A. Snyder, Distance in graphs, Czechoslovak Math. J. 1976,26 :283-296.

[15] D. J. Klein, Lukovits and I. Cutman, On the definition of the definition of the hyper-wiener index for cycle containing structures, J.Chem.Inf.Comput.Sci.1995,35:50-52.

[16] X.M. Ren, J.Y. Zou and H. Z. Ren, The Minimal Kirchhoff Index Of Theta Shape Graph, WCSE 2017:1084-1088. 